\title{
REVUE
}

\section{Anhydrose chronique du cheval et des bovidés}

ON désigne sous divers noms, dans les régions tropicales humides, une affection particulière caractérisée par la disparition de la transpiration cutanée; ce sont habituellement les chevaux importés et particulièrement les chevaux de course qui sont atteints; une maladie analogue pourrait se rencontrer chez les bovins; mais c'est surtout la maladie équine qui a été observée.

On la désigne par les expressions non sweating (qui ne sue pas); dry sweating (sueur sèche), dry coat (poil sec), anhydrose chronique, toutes expressions imparfaites parce qu'elles ne traduisent qu'un symptôme.

La maladie des chevaux "qui ne suent pas " a été rencontrée dans les régions chaudes et humides des Indes anglaises et néerlandaises (Calcutta, Rangoon, Penang, Colombo, Singapour, Trinité, etc.. et aussi en Afrique du Sud (Barnes 1938).

La maladie bovine qui est parfois identifiée à celle du cheval a été observée dans l'Inde (Stewart 1942) et en Amérique du Nord, dans la zone chaude et humide des Etats du Sud (Cates 1941).

Symptômes - Lésions. - Chez les chevaux, on voit graduellement diminuer à la fois la production de la sueur et la surface cutanée qui sue; cela débute ordinairement au niveau des reins et de la croupe. la sueur perd sa propriété d'écumer; quand la maladie est installée, l'animal demeure sec même après un exercice sévère au soleil. $\AA$ un stade avancé, les poils perdent de leur couleur; il peut se former des croûtes; il y a alors un léger prurit.

Il n'y a pas que des signes locaux; l'accélération respiratoire, l'hyperthermie accompagnent et suivent l'exercice. Si le régime reste le même, les symptômes s'aggravent et la mort survient habituellement après une course. L'hyperthermie est alors considérable; on peut noter après la mort $43^{\circ} 3$, la plus haute graduation du thermomètre (M. Crawford 1947). On peut aussi assister à la mort subite au cours d'une course.

A l'autopsie, Stewart (1938) trouve toujours de la splénomégalie sans ramollissement. Il y a habituellement gastrite catarrhale avec érosions; le foie peut être légèrement cirrhotique; il y a atrophie et dégénérescence des glandes sudoripares; cependant,
Barnes (1938) trouve les glandes sudoripares normales. Dans un cas de mort subite, Gilhyard (1944) a noté de la thrombose d'une artère coronaire.

Chez les bovins européens importés dans l'Inde (jeunes et vaches laitières) outre un poil rude, on observe de l'accélération respiratoire, la respiration se faisant par la bouche, avec une température diume atteignant $41^{\circ}$, avec baisse nocturne, et en relation avec la température atmosphérique. La plupart des animaux sont en mauvais état général.

Dans le Sud de l'Amérique du Nord, les races bovines européennes présentent, en saison chaude et humide, des symptômes analogues; les animaux sont en mauvais état le chiffre des globules rouges est très bas, la respiration accélérée et difficile; la température atteint $42^{\circ}$.

Étiologie. - L'étiologie est mal déterminée. Un fait domine : chez les chevaux comme chez les bovins, la race joue un rôle important. Daris les Indes anglaises et néerlandaises, c'est chez les chevaux anglais et australiens importés, surtout les chevaux de course, qu'on observe cette maladie; elle est rare ou inexistante parmi les chevaux arabes importés du Golfe persique; elle est rare aussi chez les animaux de races locales; cependant, d'après Macgregor, à Calcutta $6 \%$ des chevaux du pays peuvent être atteints.

Dans l'Inde comme en Amérique, les bovins atteints sont également des animaux importés. Le zébu, dans les deux régions, n'est pas ou est rarement atteint; le croisement avec les races importées également (ce qui a assuré le succès de ce croisement dans les États du Sud de l'Ámérique).

Le facteur alimentaire paraît déterminant; il se confond d'ailleurs pour bonne part avec le précédent, car si le facteur race intervient, c'est peut-être parce que les animaux importés reçoivent une alimentation spéciale (chevaux de course, vaches laitières et leurs descendants) d'où est exclu le pâturage. La maladie rétrocède quand les chevaux de course sont soumis au régime vert, qu'òn diminue l'avoine; les sueurs réapparaissent quand on les met au pâturage. L'absence de vert interviendrait en diminuant le taux des chlorures du sang (Gilhyard 1944) : chez une jument atteinte, le taux des chlorures n'est que de $248 \mathrm{mgr}$. pour $100 \mathrm{cc}$, 
au lieı de ' 420 à $480 \mathrm{mgr}$, taux normal, et l'adnuinistration de sel, en relevant le taux des chlorures du sang, fait réapparaître les sueurs.

Le'climat est la première cause invoquée : c'est toujours en zone chaude et humide que la maladie est rencontrée, et une amélioration suit aussitôt le transfert en région plus sèche et moins chaude; mais c'est le facteur humidité qui domine, car la maladie n'est pas signalée dans les pays chauds et secs. A Ceylan, on a pris l'habitude de transporter les chevaux atteints à Colombo (au niveau de la mer), dans une région montagneuse.

Pour Barnes (1938) la chaleur humide intervient en agissant sur la balanice des sels sanguins.

Une autre cause a été invoquée par Stewart (1938) : l'usage excessif de l'arsenic chez les' chevaux de courses, hypothèse qu'aurait confirmé l'analyse comparative des poils et des organes d'animaux sains et d'animaux malades.

En ce qui cóncerne les bovins, on est par ailleurs . tenté de faire un rapprochement entre la maladie qui nous occupe et les manifestations cutanées de l'avitaminose A signalées en Afrique.

Il semble en résumé que « un climat chaud et humide, une ration riche en protéine et pauvre en nourriture verte et un travail rude sont les conditions. dans lesquelles la maladie apparaît, le facteur de base étant apparemment le climat chaud et humide » (M. Crawford 1947). Mais le facteur alimentaire ne serait-il pas le plus important? De nouvelles recherches permettront seules de répondre à cette question.
Traitement. - Áux trois éléments étiologiques que nous venons de citer, correspondent des indications hygiéniques : changement de climat, changement de régime, mise au repos; la. prédominance revient au changement de régime (chez les chevaux tout au moins) : l'amélioration suit la mise au pâturage; d'ailieurs le transfert en une autre régión entraîne aussi un changement de régime.

L'abaissement du taux dés chlorures, sanguins a conduit' à prescrire l'administration de sel (Gilhyard 1944). Chez une jument qui n'avait pas: sué depuis deux ans, l'injection intraveineuse de $500 \mathrm{cc}$. d'eau salée physiologique contenant $10 \%$ de glucose a ramené l'apparition des sueurs au cours d'un galop effectué 15 minutes après l'injection; l'amélioration de l'état général fut obtenue par l'addition à la ration journalière d'une cuillerée à soupe de sel et quinze jours après, le taux des chlorures șanguins était revenu normal.

G. C.

\section{BIBLIOGRAPHIE}

1938. BARNES (J.-E.). - " Dry sweating "' chez le cheval. Veterinary Record, 30 juillet; p. 977.

1938. STEWART (C.-M.). - " Chevanx qui ne suent pas". Journ. of Royal Army Veterinary Corps, p. 161.

1941, LATES (T.-S.). - Révolution dans le Sud. Contry life, Garden City. New-York. Juillet. An. in Veterinary Bulletin, vol. 13, 434 .

1942: STEWART (C.-M) ) - Une condition du bétail des fermes laitières militaires de l'Inde ressemblant au " dry coat" ou " non sweating" des chevaux. Journ. of Royal Army" Votorinary Corps, 13, 117.

1944. GLHYARD (R.-T.). - Anhydrose chronique avec abais. sement des chlorures sanguins chez les chevaux de course. Cornell Veter, 34, 332.

1946. CRAWFORD (M.). - Analyse du travail de Gillhyard. Veterinary Bulletin, 17, 195. 